\title{
¿AFINIDAD O INTEGRALIDAD? PROCESOS DE INSERCIÓN LABORAL DE EGRESADOS TÉCNICOS EN ARGENTINA
}

Agustina Corica ${ }^{1}$

Miguel Alfredo ${ }^{2}$

\begin{abstract}
RESUMEN: La investigación indaga sobre la afinidad entre la especialidad de los estudios de nivel secundario, la integralidad de la formación y la trayectoria ocupacional a diez años del egreso. El enfoque fue de corte longitudinal donde se realizaron entrevistas retrospectivas a jóvenes egresados de la educación secundaria técnica de cuatro jurisdicciones de Argentina: Ciudad y Gran Buenos Aires, Neuquén y Salta. Se presentan resultados preliminares relevados en 2018. Se reconstruyen las trayectorias formativas-ocupacionales de jóvenes que egresaron en el 2006 de la escuela secundaria. Del análisis surge que la formación recibida habilita distintas inserciones laborales pero las posibilidades están fuertemente mediatizadas por la localización geográfica, por el sostenimiento familiar y por la influencia de los estereotipos de género tradicionales que remarcan y limitan cambios de rumbos y oportunidades laborales distintas.
\end{abstract}

Palabras-clave: Educación secundaria. Educación técnica. Mercado de trabajo. Inserción laboral. Jóvenes.

\section{AFFINITY OR INTEGRALITY? LABOR INSERTION PROCESSES OF TECHNICAL GRADUATES IN ARGENTINA}

\begin{abstract}
The research aims to analyze the affinity between the specialty of secondary level studies, the integrality of training, and the occupational trajectory ten years after graduation. The approach was longitudinal, in which retrospective interviews were conducted with young graduates of technical secondary education in four jurisdictions in Argentina: Ciudad and Greater Buenos Aires, Neuquén, and Salta. Preliminary results are presented in 2018. The training-occupational trajectories of young people who graduated in high school in 2006 are reconstructed. From the analysis it appears that the training received enables different labor insertions, but the possibilities are strongly mediated by geographical location,
\end{abstract}

\footnotetext{
El artículo surge de la investigación titulada "La Construcción de Trayectorias Laborales de los Egresados de la Escuela Técnica: Una Mirada sobre la Integralidad de la Formación a una Década de la Ley de Educación Técnico Profesional”, cuya coordinación estuvo a cargo de la Dra. Ana Miranda y contó con financiamiento del Fondo Nacional de Investigaciones de Educación Técnico-Profesional, dependiente del Instituto Nacional de Educación Tecnológica (FONIETP-INET).

1.Facultad Latinoamericana de Ciencias sociales - Programa Juventud y Universidad Pedagógica Nacional - Buenos Aires, Argentina. E-mail: acorica@flacso.org.ar

2.Facultad Latinoamericana de Ciencias sociales - Programa Trabajo - Buenos Aires, Argentina. E-mail: malfredo@flacso.org.ar

Editor de Sección: Salomão Barros Ximenes
} 
family support, and the influence of traditional gender stereotypes that highlight and limit changes in different directions and job opportunities.

Keywords: Secondary education. Technical education. Labor market. Labor insertion. Youth.

\title{
AFINIDADE OU INTEGRALIDADE? PROCESSOS DE INSERÇÃO TRABALHISTA DE GRADUADOS TÉCNICOS NA ARGENTINA
}

\begin{abstract}
RESUMO: A pesquisa investiga a afinidade entre a especialidade dos estudos de nível secundário, a integralidade do treinamento e a trajetória ocupacional dez anos após a graduação. A abordagem foi longitudinal, na qual foram realizadas entrevistas retrospectivas com jovens graduados do Ensino Médio técnico em quatro jurisdições da Argentina: Cidade e Grande Buenos Aires, Neuquén e Salta. Os resultados preliminares são apresentados em 2018. As trajetórias de formação profissional dos jovens que se formaram no Ensino Médio em 2006 são reconstruídas. A partir da análise, parece que o treinamento recebido possibilita diferentes inserções trabalhistas, mas as possibilidades são fortemente mediadas pela localização geográfica, apoio familiar e influência dos estereótipos tradicionais de gênero que destacam e limitam as mudanças em diferentes direções e oportunidades de trabalho.
\end{abstract}

Palavras-chave: Ensino Médio. Educação técnica. Mercado de trabalho. Inserção do trabalho. Juventude.

\section{La Relación Educación y Trabajo: Transiciones. Debates Clásicos y Actuales}

$\mathrm{E}$ $\mathrm{n}$ la actualidad existe consenso respecto a que luego de las transformaciones sociales vinculadas con la crisis de la sociedad salarial, las trayectorias educativas y laborales perdieron el carácter lineal. En este sentido, cada vez es más difícil predecir con suficiente certeza el futuro a partir del origen familiar, como sucedía en la sociedad industrial, pero tampoco el capital humano parecería estar funcionando como predictor de la posición en la estructura social (GIL CALVO, 2009). Es por eso por lo que estudios recientes mencionan que cada vez menos los jóvenes realizan trayectorias de inserción lineales y cada vez más las inserciones laborales adquiere diversos itinerarios que muchos de ellos son de carácter precarios y cambiantes (CASAL et al., 2006). Por otro lado, como consecuencia de los cambios tecnológicos, se han dado cambios significativos en la organización de la producción y del trabajo. Estos conllevan nuevos requerimientos de competencias, a veces difícilmente adquiridas a través de la educación formal y, sobre todo, una gran inestabilidad e incertidumbre acerca de las competencias, saberes y habilidad requeridos en el mediano y largo plazo. Paralelamente, se destaca los procesos de devaluación de las credenciales educativas adquiridas en el sistema de educación formal, a pesar de que continúan siendo importantes para acceder al mercado de trabajo, ya no garantizan el acceso a mejores empleos (COLL, 2011).

En relación con esto, algunos estudios destacan que existe una disminución del efecto de los diplomas en el requerimiento de los puestos laborales disponibles. Otros señalan que más allá de que la educación ya no garantiza los puestos de trabajo según el diploma escolar obtenido, la educación sigue siendo indispensable, en donde la posibilidad de oportunidades se encuentra íntimamente vinculada con las 
credenciales, sea como limitante o bien como marco para la competencia (BAUDELOT; LECLERCQ, 2008). Es decir, las certificaciones educativas siguen siendo relevantes, pero no suficientes (FILMUS et al., 2001). En este marco de situación, la competencia entre titulaciones hace que se desdibuje el peso de la educación como igualadora de las oportunidades laborales. Investigaciones recientes sobre la inserción laboral juvenil han evidenciado que, si bien el problema del desempleo y precariedad juvenil es de magnitud relevante, la causa de ello se encuentra más asociada al propio mercado de trabajo y a la estructura social (WELLER, 2003).

De este modo, los debates críticos clásicos hay concentrados dos grandes posturas que dan cuenta del vínculo sobre la adecuación de los conocimientos adquiridos en el sistema educativo y los requerimientos del mercado de trabajo. Por un lado está el enfoque adecuacionista o teoría de la correspondencia (BOWLES; GINTIS, 1986; WILLIS, 1988) que demuestra la autonomía relativa del sistema educativo respecto a las demandas del mercado de trabajo, señalando que la demanda de niveles formativos responde a una lógica relativamente independiente de los requisitos de la demanda de trabajo en términos de cualificación profesional y que un enfoque basado en las competencias laborales permite analizar e interpretar mejor las relaciones entre formación y empleo (COLL, 2011).

Este enfoque comenzó a perder peso cuando las teorías criticas reproductivistas demostraron que los jóvenes presentaban bajos niveles de correspondencia entre especialidad de formación y especialidad del puesto de trabajo ocupado, asociándose con los fenómenos de desempleo y sobre-educación. Sin embargo, otras investigaciones demuestran que en algunos trabajos la adecuación entre especialidad de formación y empleo continúan siendo relevante, especialmente entre aquellas que las "licencias profesionales" son requeridas para ser ejercido esas ocupaciones (BÉDUWÉ et al., 2005). Pero estas dos miradas dan cuenta de una tensión entre la adecuación de formación y empleo que se inscribe en los debates sobre el vínculo educación y trabajo más amplios. En este sentido, se asocia que esta relación se complejiza cuando en el análisis se incorpora la variable social que demuestra una vez más que la relación entre educación y trabajo, entre credenciales educativas y puestos de trabajo, así como niveles de escolaridad y niveles de ingresos son espurios y están condicionados por una multiplicidad de factores que intervienen y determinan rumbos diversos. El sector social, la estructura productiva, la rama de actividad y el contexto sociopolítico influyen en esta relación dando un carácter más inespecífico, desigual, complejo y heterogéneo.

No obstante, y sin desconocer los aportes de estas corrientes clásicas, diversos debates actuales han referido a que la linealidad propuesta por los esquemas clásicos, aunque valiosos en términos analíticos, resultan insuficientes a la hora de comprender la tríada jóvenes-educación-trabajo y como esta configura y habilita a la vida adulta en los tiempos contemporáneos.

A partir de los cambios producidos en el régimen de acumulación, las investigaciones occidentales han puesto mayor énfasis en aspectos coyunturales del orden socioeconómico y político para comprender las transiciones juveniles. La incorporación de la temporalidad y territorialidad en clave contextual permiten un mayor marco de comprensión a los procesos de reflexividad e individuación propio de Occidente (BENDIT; HAHN-BLEIBTREU, 2008). Estos procesos ponen de relieve la centralidad que adquieren las biografías juveniles, las cuales se presentan como construcciones cotidianas entramadas en la vulnerabilidad social imperante. En este sentido, el lugar de la construcción biográfica de la juventud confiere un lugar central a la temporalidad y territorialidad coyuntural, en donde las condiciones efectivas sobre las posibilidades de empleo requieren retomar la reflexividad como espacio de agencia en la construcción de identidades segmentadas (FURLONG, 2009; CUERVO; WYN, 2014; REYNOLDS, 2015).

El carácter de interseccionalidad clase-género-edad incorporado por la noción de construcción biográfica en relación con la reflexividad, inserción tempo-territorial y estructura, cobro un núcleo central en los estudios de juventud con base en la sociología. Bajo la noción de "generación social" (WYN; WOODMAN, 
2006), surge la noción de "gramáticas de la juventud". En términos analíticos, la gramática de la juventud apuesta al estudio situados de los espacios socialmente validados para las experiencias juveniles en sus diversos ámbitos, lo cual se conjuga con la capacidad de agencia que estos jóvenes tienen al momento de resignificar las determinaciones inter e intrageneracionales (MIRANDA; CORICA, 2018). Esta vinculación entre agencia y estructura ha dado un campo fecundo a los estudios de juventud bajo la forma de transición(es) juvenil(es).

No obstante, el estudio del vínculo educación-trabajo en Argentina no ha permanecido inmutable a estas tendencias analíticas, donde todo comienza a cambiar a partir de la obligatoriedad del nivel medio. La educación secundaria amplía sus funciones sociales dejando de ser un nivel terminal el cual habilitaba puestos de trabajos específicos para pasar a ser un nivel mínimo obligatoria para ingresar al mercado de trabajo, perdiendo su correspondencia directa con un puesto laboral. Sin embargo, la escuela media técnica históricamente en Argentina fue "fundada" con una mira en la salida laboral según la especialidad de su oferta que las otras modalidades como son el bachiller y el comercial (aunque este si fue vinculado apuestos laborales específicos) orientaciones del secundario donde existía una función más propedéutica que de formación para el trabajo. En este sentido, analizar el vínculo educación y trabajo en la educación secundaria técnica implica pensar en el rol que juega la especificidad de las orientaciones de esta modalidad educativa en las trayectorias laborales de los y las jóvenes. Esta línea de estudios señala que es la especialidad de la formación y no el nivel lo que juega un rol significativo en la relación (BORRAS et al., 2008). Por eso, el presente texto tiene como uno de sus objetivos, profundizar en el análisis de este vínculo centrándose en la modalidad técnica del nivel medio de enseñanza.

Ahora bien, de manera de vincular los debates clásicos con los más contemporáneos es que se analiza las trayectorias de jóvenes egresados de escuelas secundarias técnicas de distintas jurisdicciones del país en el marco de distintas transformaciones que se fueron sucediendo en Argentina a fines del Siglo XX. La debacle económica que se produjo a nivel local en el 2001 y las transformaciones en la estrategia económica implementada a partir del 2003 acompañó un proceso regional caracterizado por un crecimiento económico. En el plano educativo, la educación técnica volvió a alcanzar gran protagonismo, junto con la sanción de la Ley de Educación Técnico Profesional (n. 26.058/05), y el desarrollo de acciones de promoción de esta modalidad. Esto junto con la sanción de la Ley de Educación Nacional n. 26.206/06 (en adelante LEN) que define la obligatoriedad de la educación secundaria, y en años siguientes la Asignación Universal por Hijo en 2009, y el Programa PROGRESAR en el 2014 signaron un marco de derechos en donde el Estado Nacional asumió un fuerte compromiso en la promoción de las condiciones de vida de los y las jóvenes en nuestro país (KESSLER, 2014). Es este punto que el estudio realizado quiere dar cuenta de los elementos y factores que influyen en los procesos de construcción de las trayectorias laborales de egresados de escuelas técnicas de Argentina. De este modo, las preguntas problema central fueron: ¿Cómo son las trayectorias educativas y laborales de jóvenes egresados/as del secundario técnico de distintas regiones de Argentina? ¿Qué factores y/o elementos influyeron y/o posibilitaron los procesos de inserción laboral juvenil? ¿Qué relación existe entre la formación recibida y la inserción laboral lograda? ¿Qué vinculo se da entre la especialidad técnica y las tareas desarrolladas en sus puestos de trabajo?

En la presente investigación se debate la relación entre la formación técnica de nivel medio y la inserción laboral de jóvenes egresados con una primera hipótesis que sostiene que no es posible proponer una función económica universal y predeterminada de la educación respecto del mercado de trabajo, sino que esta función solo puede ser investigada a través del análisis de situaciones socio históricas concretas. Y una segunda hipótesis, asociada a la educación técnica que sostiene que sobre principios de siglo veintiuno las características de la organización productiva y de los mercados de trabajo generan que los procesos de inserción laboral de los y las jóvenes se dé de forma cada vez más prolongada. El artículo presenta hallazgos 
de una investigación realizada en el marco del Programa de Juventud de la FLACSO Argentina, el cual centra su actividad en los estudios del proceso de inserción laboral a través de la aplicación de investigaciones sobre la transición educación-trabajo.

\section{Abordaje Metodológico}

La investigación se realizó con un abordaje metodológico de corte longitudinal utilizando la técnica de entrevista biográfica retrospectiva. De corte cualitativo, el enfoque retomado es el método biográfico, con base en la tradición de la escuela de los "curso de vida" (ELDER, 1994). Dicho método investiga la narrativa biográfica como expresión de un conjunto de experiencias y sucesos (SAUTU, 2004), en donde el despliegue de experiencias a lo largo del tiempo se enmarca en contextos determinados atravesados por múltiples relaciones sociales e instituciones (familia, escolaridad, empleo), con un epicentro en la selectividad del relato enunciado por el protagonista.

La construcción del panel se desarrolló a partir de entrevistas biográficas retrospectivas, mediante la técnica tracer studies de seguimiento de egresados, que releva información de forma posterior al egreso. La unidad de análisis son mujeres y varones que egresaron de la educación técnica en el año 2006, de escuelas localizadas en Ciudad de Buenos Aires, Provincia de Buenos Aires, Neuquén y Salta.

En base a la producción de información cualitativa, se realizó un estudio exploratorio acerca de las trayectorias sociales de jóvenes. La muestra fue realizada de manera selectiva, en base a criterios teóricos (GLASER; STRAUSS, 1967). Así se definieron los criterios para la realización de la muestra, diseñada a partir de las variables jurisdicción, género y especialidades educativas. Se abordó la problemática de las trayectorias juveniles en un análisis situado, tomando la generación de individuos nacidos entre 1987 y 1989 , que al momento de las entrevistas tenían entre 29 y 31 años. La muestra estuvo compuesta por un total de 69 jóvenes que egresaron de la educación técnica en el año 2006. El trabajo de campo fue desarrollado en las jurisdicciones durante los meses de abril y junio de 2018.

Es necesario señalar que también se ajustó a las posibilidades existentes en las distintas jurisdicciones donde se realizó el trabajo de campo, de forma de abarcar todas las orientaciones de las escuelas técnicas y considerar una cantidad homogénea entre provincias. El reclutamiento de los/as entrevistados/as se realizó por medio de contactos informales. Esta decisión redundo en la utilización de la técnica "bola de nieve" dentro de las entrevistas a realizar para los/as egresados/as de la misma escuela. Esta permitió, por medio de los contactos suministrados por los propios egresados/as, alcanzar el número de jóvenes definidos previamente ${ }^{1}$.

En cuanto a la investigación realizada y a partir de los datos cualitativos relevados se procedió a analizar la evidencia empírica construyendo tipologías de las trayectorias educativo-laborales de los y las jóvenes egresados de la escuela media técnica (cohorte 2006) de las cuatro jurisdicciones que abarco el estudio. Las mismas fueron construidas a partir de la situación de los jóvenes al final de los recorridos. Los criterios considerados son por un lado la integralidad educativa, dividiendo a las trayectorias entre "integrada" y "no integrada”. Por otro lado, se analiza el tipo de trabajo actual y su vinculación con la especialidad de la escuela secundaria de la cual egresaron. En cuanto a las trayectorias educativa integradas son aquellas trayectorias de continuidad de estudios postsecundarios relacionados con la especialidad de la escuela y se incluye los distintos niveles educativos: formación profesional, terciario, universitario. En cambio, en las trayectorias "no integradas" comprende a los y las jóvenes que no continuaron estudiando tanto como a quienes realizaron recorridos de estudios en áreas no relacionadas con la especialidad de la escuela. En los casos de jóvenes que habían abandonado los trayectos educativos postsecundarios se tomó la decisión de considerar como 
trayectorias integrada sólo si el joven había realizado más de la mitad del trayecto. En cuanto a las trayectorias laborales, se clasifican como trayectorias vinculadas a aquellas en las que hay relación entre la especialidad de la escuela y el trabajo realizado en el momento de la entrevista. Y trayectorias laborales desvinculadas a aquellas en las que los y las jóvenes realizan ocupaciones no relacionadas o no realizan actividades productivas remuneradas (Tabla 1).

Tabla 1. Tipologías de trayectorias educativo-laborales

\begin{tabular}{cc}
\hline Tipologías & Trayectorias \\
\hline Tipo I & Trayectoria educativa integrada y trayectoria laboral vinculada \\
\hline Tipo II & Trayectoria educativa no integrada y trayectoria laboral vinculada \\
\hline Tipo III & Trayectoria educativa integrada y trayectoria laboral desvinculada \\
\hline Tipo IV & Trayectoria educativa no integrada y trayectoria laboral desvinculada
\end{tabular}

Fuente: Elaboración propia.

A partir de la tipología construida se analizan puntualmente los distintos cursos de formación que realizaron los jóvenes a lo largo de la década ("recorrido de estudios postsecundarios"), su relación con la especialidad de la escuela secundaria y los motivos por detrás de la elección de los cursos o carreras postsecundarios. Además, se indaga en la opinión de los jóvenes sobre la escuela técnica para poder comprender los factores que influyen en las decisiones que tomaron los jóvenes luego del egreso. Se examinaron los aportes tanto de los conocimientos aprendidos como del título obtenido, y las opiniones sobre la influencia del recorrido secundario en la elección de los estudios postsecundarios. También se relevan las experiencias laborales que los jóvenes tuvieron mientras cursaban la escuela secundaria para conocer cómo fueron las primeras inserciones. Interesan también los contactos laborales que los llevaron a ingresar en los empleos para dar cuenta de las distintas formas de acceso al trabajo.

La selección de la muestra se basó a partir de criterios teóricos de una muestra segmentada por establecimientos educativos de carácter intencional y no probabilística. Esta definición se retomó a partir de criterios tradicionales dentro de los estudios de segmentación educativa y con un amplio campo de relevancia empírica por parte de la sociología de la educación (MIRANDA; CORICA, 2018). La muestra de establecimientos educativos quedo compuesto por un total de 29 escuelas, las cuales se distribuyeron de la siguiente manera: nueve escuelas de CABA, siete escuelas de Provincia de Buenos Aires, cuatro escuelas de Neuquén y nueve de Salta. Las dimensiones recabadas para la incorporación a la muestra fueron: localización, infraestructura, institución, cuerpo docente y sector social predominante de la población que asiste. Si bien se codificaron las referencias institucionales para mantener la confidencialidad debida, en el Tabla 3 del Anexo se presenta el universo y distribución según la caracterización seleccionada. La distribución De las trayectorias identificadas es la siguiente:

Tabla 2. Composición de la muestra según tipología

\begin{tabular}{cccc}
\hline Tipología & Varón & Mujer & Total \\
\hline Tipo I & 27 & 10 & 37 \\
\hline Tipo II & 2 & - & 2 \\
\hline Tipo III & 6 & 9 & 15 \\
\hline Tipo IV & 6 & 9 & 15 \\
\hline Total & 41 & 28 & 69 \\
\hline
\end{tabular}

Fuente: Elaboración propia. 
Ahora bien, las diferencias están en la composición por jurisdicción. En el tipo IV, la mayoría es de Salta. En cambio, en el tipo III la distribución por jurisdicción de los casos es bastante homogénea. Por último, el tipo II es el grupo menos numeroso (sólo dos casos). Compuesto por varones, uno de NQN y otro de SLA. Por su parte, el tipo I se encuentra presente en todas las jurisdicciones (Tabla 2).

Las condiciones de acceso y permanencia al mercado laboral presentan en Argentina una fuerte segmentación regional y por rama de actividad para la población juvenil. En este sentido, el ingreso, estabilidad y calidad del empleo son diferenciados entre las provincias a raíz de las propias realidades en las cuales se insertan (PÉREZ; BUSSO, 2018). Con una estructura económica primarizada, la centralidad de las actividades de explotación agraria de enclave con baja o moderada incorporación tecnológica es la realidad sobre la que insertan las jurisdicciones de Salta y Neuquén, mientras el área del CABA y Conurbano Bonaerense incorpora un mayor nivel de industrialización y actividades vinculadas al turismo, con un aglomerado de población significativamente superior (KEOGAN et al., 2020). En el siguiente apartado se analiza las trayectorias laborales de los y las egresados considerando las distintas tipologías identificadas, como se mencionó, algunas presentan mayor afinidad e integralidad y otras menor vinculaciones con el título secundario técnico obtenido.

\section{Trayectorias Laborales de Egresados de Industrial y Servicios. Entre la Afinidad y la Integralidad}

Según las ideas centrales planteadas se destacan que, en términos generales, de las trayectorias identificadas, la mitad de los entrevistados se encuentran en las trayectorias educativas integradas y laborales vinculadas. Trayectorias que dan cuenta de la importancia y relevancia del título técnico. También el escaso número de recorridos donde hay vinculación laboral pero no integralidad educativa, pone en evidencia la importancia de los estudios postsecundarios. Por otro lado, se destaca que en el caso de los egresados y egresadas de la jurisdicción de Salta es donde se concentran la mayoría de los casos con menos trayectorias integradas y vinculadas.

En la tipología II, que es la menos significativa ya que sólo se identificaron dos casos, son jóvenes varones uno de Salta y otro de Neuquén que no continuaron estudios postsecundarios, pero sí realizaban trabajos relacionados con la especialidad del secundario. Este tipo de trayectorias se debería profundizar en un estudio específico para dar cuenta de las características de este grupo. Diego, uno de los jóvenes, refleja en su historia la falta de disponibilidad económica por parte de su familia, lo que llevo a retirarse de la carrera universitaria que había iniciado para incorporarse al mercado laboral:

Mis padres me acompañaron mucho en las técnicas porque la verdad que ellos tenían que darme todos los días plata para comer porque básicamente yo vivía ahí, pero ya cuando llegue a la UNSA ellos optaron por hacerse a un lado y preguntaron... o estudiás o trabajás [...] todos tenemos diferencias en la familia y bueno ellos no tenían para sustentarme asique me dijeron que empiece a trabajar... empecé a trabajar y no me daban los tiempos lamentablemente no me daban los tiempos... (E56-V-SLA-SERVICIO).

Sin embargo, en 2009 Diego se incorpora al ejército como soldado, situación que, mediante su título en Técnico en Administración de Empresas, luego pasa a trabajar como oficinista civil, empleo que mantenía al momento de la entrevista. 
En el caso de las trayectorias tipo III (educativa integrada y laboral desvinculada) se encuentran recorridos que evidencian diversas situaciones por las cuales luego del egreso y de estudiar carreras relacionadas con la especialidad de la escuela, los jóvenes no se encuentran insertos laboralmente en empleos relacionados al finalizar la tercera década de vida. Algunos se encontraban realizando empleos en otros rubros porque no encontraban demanda laboral de calidad en sus jurisdicciones. Este es el caso de Pedro, quien había realizado un secundario con orientación tecnológica y mecánica del sur del Conurbano Bonaerense y al egresar realizó diversos cursos profesionalizantes integrados con la especialidad (como técnico reparador de PC y otros cursos de redes). Relataba:

[...] estudie la carrera y salí de ahí ya con la idea de querer mezclar estas dos cosas, la tecnología como un canal de información para la gente que hoy todo el mundo está conectado a dispositivos móviles, computadoras, tabletas, pero trasmitir estos trabajos en lo que tiene que ver con el cuidado del medio ambiente, lo que hacen los guarda parques, hoy en día entrevisto gente y me manejo con la tecnología para trasmitir estas cosas (E8-V-PBA-INDUSTRIAL).

En los primeros años de la trayectoria laboral le sirvieron sus conocimientos relacionados con la especialidad de la escuela, ya que se insertó en empresas realizando soporte técnico de equipos y redes. Al finalizar la tercera década, y una vez recibido de su formación terciaria, se insertó laboralmente con su nueva profesión (trabajaba de guarda parques y de periodista en temas ambientales).

Para otros jóvenes se debía a que aún no habían finalizado los estudios postsecundarios, por lo que creían que en un futuro podrían encontrar empleos de su profesión. Es el caso de Julia (E58-M-SLASERVICIO), quien egreso de una escuela de un barrio relegado del sur de la Ciudad de Salta con el título de técnica en informática. Con una primera inserción en diversos establecimientos de atención al público, y luego de un paso por la carrera de Recursos Humanos, comenzó el profesorado de matemáticas, estudios que sustenta con un emprendimiento de pastelería propio.

Y, por otro lado, están las trayectorias del tipo IV que se diferencias en los recorridos postsecundarios. Algunos estudiaron carreras o cursos en áreas diferentes a la de la especialidad de la escuela y otros no continuaron ningún estudio luego de egresar. Pero en ningún caso los y las jóvenes se encontraban al final del recorrido en empleos que evidenciaran vinculación con la especialidad técnica. En este grupo hay tanto varones como mujeres de las distintas especialidades. Claudia (E36-M-NQN-INDUSTRIAL), egresada como técnica en química, proveniente de un barrio postergado de los alrededores de la Ciudad de Neuquén, contó con una temprano incorporación laboral mientras cursaba la secundaria, realizando diversos trabajos temporales vinculados al cuidado de niños y limpieza. Luego del egreso su recorrido laboral oscilo entre unas primeras experiencias en un laboratorio geoquímico, para pasar a otros rubros vinculados a los servicios en pos de mejoras salariales. Al respecto de su recorrido postsecundario ella comenzó la carrera de Biología, no obstante, acusaba su discontinuidad por falta de recursos económicos y disponibilidad horaria de la oferta

[...] por varias cuestiones, o sea, en la universidad, en Mendoza hay una realidad y que la Universidad de Cuyo es una las universidades más exigentes que hay a nivel nacional, pero no solamente por el contenido que te exigen estudiar sino que ellos no piensan en que la mayoría de las personas que están estudiando no siempre van a tener la posibilidad de que los padres o algún familiar le pague los estudios [...] había materias que yo no las podía hacer porque se dictaban en la tarde y no había comisiones en la mañana porque ellos no querían [...] (E36-M-NQN-INDUSTRIAL). 
En cuanto a las jurisdicciones, se observa una mayor cantidad de jóvenes provenientes de la jurisdicción analizada del norte del país, Salta. En cuanto a las trayectorias educativas, no tuvo peso la escuela secundaria a la hora de elegir una carrera o curso para continuar los estudios. Y, en este sentido, se destaca que quizás esto tenga que ver con que la oferta de carreras postsecundarias ofrecidas en la jurisdicción fue un factor que influyó en los recorridos. En algunas jurisdicciones las posibilidades para continuar estudios estaban dentro del ámbito privado por lo que fueron opciones dificultosas para aquellos que no contaron con recursos de sostén familiar. Es el caso de Juan (E66-V-SLA-INDUSTRIAL), egresado de la especialidad en bienes y servicios con orientación en automotores, quien inició carrera de Criminalística en la Universidad Católica de Salta, la cual no pudo finalizar. Con respecto a su trayectoria laboral, si bien los saberes adquiridos en la formación media resultaron útiles para incorporarse a actividades de mantenimiento, los sucesivos empleos (vinculados al sector comercial) demostraron un recorrido disperso.

Ahora bien, en la tipología I, la más exitosa o esperable, se encuentra un grupo de jóvenes egresados y egresadas que lograron integrar su formación postsecundaria con la especialidad de su título técnico y obtuvieron una inserción laboral vinculada con esa formación. Se caracteriza por la heterogeneidad de tipos de formación, la multiplicidad de experiencias educativas (tienen diversas formaciones, aunque logren sólo finalizar una) y diversidad de experiencias laborales que en algunos casos se fueron hilvanando hasta alcanzar el trabajo que tenían al momento de la entrevista. En el caso de las mujeres, si bien, la mayoría continuó estudiando carreras universitarias vinculadas a su especialidad y trabajan de eso actualmente, también se destacan distintas experiencias formativas de corta duración (cursos, carreras terciarias). Eso hizo que muchas de ellas permanezcan más años en el sistema educativo y por lo tanto les llevó más tiempo terminar la carrera universitaria (muchas de ellas se recibieron entre el 2015-2017). Clara, una joven de CABA que se recibió con un título en Diseño de Interiores contaba:

Sí, egresé de la escuela y ese año estuve también haciendo cursos presenciales en la UP (Universidad de Palermo) relacionados con el diseño. Eran cursos por ahí de uno o dos días, gratuitos, por ahí de temas específicos, podía ser de iluminación o algún otro tema específico (E02-M-CABA-SERVICIOS).

Clara finalizó la carrera universitaria de arquitectura y en los últimos años de su trayectoria había realizado distintos cursos cortos en temas específicos, según contaba, complementarios con su carrera universitaria. También realizó una especialización en Seguridad e Higiene en la construcción en la Universidad de Buenos Aires. La entrevistada destacaba la necesidad de obtener el título universitario para tener "el plus" que ella quería alcanzar:

\footnotetext{
Me pasó que hice diseño de interiores, y me faltaba un plus para decir "bueno, listo, yo quiero diseñar esto, y esta pared la tiro”. No... como diseñadora, no podés, tenés que ser arquitecta, tenés que hacer... para el plus que yo quería, necesitaba tener una carrera, tenía que tener más conocimiento. Entonces, seguí arquitectura. (En la escuela) tenía muchos profesores arquitectos también (E02-M-CABA-SERVICIOS).
}

El caso de Clara es un ejemplo de los recorridos de jóvenes mujeres en los que se dio una simultaneidad de distintos tipos de formación con la actividad laboral. La multiplicidad de actividades da cuenta de una de las formas que asumen los recorridos educativos y que están por detrás del alargamiento en el tiempo de la cursada de la carrera de nivel superior, por lo que logran finalizar los estudios diez años después de haber egresado. Este es el caso de Inés, egresada del secundario técnico de la jurisdicción PBA con 
especialidad en electrónica, quien se había recibido en el año 2017 de Ingeniera en Sistemas de Información en la Universidad Tecnológica Nacional ubicada (CABA). Además de la carrera ella había hecho otros estudios: una especialización en "Experto universitario en supervisión”, curso en "Desarrollo modal”, y cursos sobre herramientas de lenguajes y experiencia en el área de programación y aplicaciones. En la actualidad se encontraba cursando otra especialización en Ingeniería en telecomunicaciones, en la misma universidad.

En otros casos, el alargamiento del recorrido y la permanencia en el sistema educativo se da por cambios de carrera o de instituciones. Es el caso de Jésica, una joven de la Ciudad de Neuquén egresada de la especialidad Maestro Mayor de Obra, quien presentó un recorrido educativo alargado en el tiempo y con tiempos de espera. Ella obtuvo el título secundario técnico dos años después de haber terminado de cursar, cuando logró rendir las materias previas que le habían quedado. Esta entrevistada no siguió una carrera universitaria, pero si cursos de formación profesional vinculados a su especialidad. Jesica contaba:

En el 2008, empecé a recursarlas y saqué todas las materias menos una que me quedó. En esa fecha ya mi papa trabajaba acá en el hospital y me dijo que faltaba gente y me llevó para que concurse. Y me presenté y desde ese entonces trabajo acá. Una vez que ingresé al hospital, empecé a recursar algunas materias y me recibí en el 2012 más o menos (E42-M-NQN-INDUSTRIAL).

En el segundo tiempo de su trayectoria laboral, Jesica se tomó un "año sabático", para retomar posteriormente cursos de formación profesional (curso de gasista, de Autocad y de termo fusión), los cuales le aportaron a su inserción laboral en la que realizaba tareas vinculadas.

Por otro lado, en estas trayectorias tipo I se encuentran empleos que en su mayoría son de inserción profesional, y en su minoría son en trabajos informales, precarios y familiares (en el caso de los varones de especialidad agraria) así como emprendimientos propios. Esto son los casos de egresados que iniciaron trayectos universitarios que no lograron finalizar, pero luego de idas y vueltas acumularon distintos tipos de formación: carreras terciarias y cursos de formación profesional. Sin embargo, estas experiencias educativas no finalizadas les dieron herramientas para poner en marcha un emprendimiento propio o realizar trabajos de forma autónoma e independiente. Algunas de las egresadas realizaron emprendimientos relacionados con la carrera terciaria que realizaron. Uno es el caso de Lucía, una entrevistada de la jurisdicción CABA, egresada en el secundario especializado en Diseño de Interiores. En su recorrido educativo ella inició diversas carreras (Arquitectura, Perito Calígrafo, Organización de Eventos), y finalmente terminó el último de los trayectos terciarios. Según analizaba Lucía, su elección estaba en relación con la escuela:

[...] Yo quería ser arquitecta, no quería ser diseñadora de interiores. Quería ser arquitecta. Entonces, a mí la parte que más me gustaba era la parte estructural, El estudio de la parte estructural. Y cuando empecé arquitectura y después la dejé, [...] busqué a ver qué era lo segundo que más me gustaba: organización de eventos. No tenía nada que ver, pero... hoy soy organizadora de eventos. [...] Para esto sí me sirvió un montón el título, ahora que lo pienso. Porque yo tuve ambientación y muchas cosas del color y qué sé yo para los eventos, y para esto sí me sirve (E07-M-CABA-SERVICIO).

Dentro de este tipo de recorrido, otras mujeres presentan trayectorias de inserción vinculadas con actividades sociales y educativas, y que destacaron que el titulo técnico les dio conocimientos y habilidades para desempeñar estas tareas. Especialmente estos recorridos se dan en las mujeres que viven en las jurisdicciones Salta y Neuquén, que no han podido terminar la carrera universitaria por problemas económicos o por cuestiones familiares y que realizaron cursos de formación profesional vinculados con la especialidad de la 
escuela secundaria. En el caso de Ivana, una de las egresadas de Salta de la especialidad Danzas Folclóricas, su inserción laboral estuvo siempre vinculada con áreas sociales y educativas (E68-M-SLA-SERVICIO).

Los egresados varones que desarrollaron trayectorias integradas y habían logrado la vinculación presentan rasgos comunes entre las jurisdicciones abordadas. Esencialmente, estos jóvenes coincidían en que los saberes y contenidos adquiridos en la educación media les habían sido de ayuda al momento de encontrar empleo. También la implementación de sus conocimientos les había resultado necesaria para llevar a cabo los mismos. Un ejemplo de eso es Carlos (E27-V-PBA-INDUSTRIAL), de una escuela industrial de la Ciudad de la Plata, ubicada en la Provincia de Buenos Aires. Egresado del secundario como Técnico en equipos e instalaciones electromecánicas, realizó la carrera de Diseño Industrial y siempre trabajó de su especialidad. Durante los primeros años, mientras estudiaba, realizaba trabajos de manera independiente diseñando diversas piezas industriales y una vez recibido lo combinó con la docencia universitaria. En la actualidad trabajaba de manera formal como proyectista en una empresa de ingeniería.

Entre los jóvenes varones egresados de la especialidad industrial, algunos se insertan como técnicos en empresas con empleos formales. En cuanto a la forma de acceso al trabajo, los consiguen a través de redes familiares y conocidas, o través de anuncios de búsquedas laborales. Según sus percepciones, es gracias a su título secundario y en conjunto a la experiencia laboral previa que consiguen este tipo de trabajos. Es el caso de Ramiro, un joven egresado de una escuela de la CABA con el título de Maestro Mayor de Obras. El joven contaba que trabajaba en una empresa de telecomunicaciones y destacaba que la carrera le había dado las herramientas para desempeñarse en este trabajo:

[...] Pero bueno, entré ahí y me gustó muchísimo el sector, digamos; el sector de comunicaciones, que es como super dinámico. Y me quedé, y me fui como desarrollando dentro de la empresa.

[...] Entonces, cada vez que me toca trabajar en eso y en el boceto de una aplicación, en la experiencia del usuario -que se llama-, ahí puedo aplicar el conocimiento, digamos. Ahí es donde más se aplica (E03-V-CABA-INDUSTRIAL).

En cuanto a la modalidad de los empleos que actualmente tienen los jóvenes, se presenta el grupo de los que hicieron emprendimientos. Así, estos jóvenes estudiaron en especialidades industriales y tuvieron recorridos postsecundarios con estudios terciarios o universitarios, destacándose la inserción laboral en ámbitos vinculados con sus orientaciones. Es el caso de Fernando, quien había realizado el secundario en la Ciudad de La Plata, egresando como Maestro Mayor de Obras. En su recorrido estudió arquitectura y tuvo experiencias laborales en estudios de arquitectura. Fernando en la actualidad contaba:

Trabajo en un estudio de arquitectos... Somos cuatro los que integran el estudio y hay una parte de dirección de obra, por ahí ir a la obra y verificar que se esté haciendo todo como se tiene que hacer, pero principalmente en la parte de proyectos... (E26-V-PBA-INDUSTRIAL).

También está el caso de Emanuel, quien una vez obtenido el título secundario de Maestro Mayor de Obras en la ciudad de Neuquén inició la carrera de ingeniería, aunque no la finalizó. Desde el primer tiempo realizaba trabajos para un arquitecto y en el momento de la entrevista ya era parte del estudio:

Soy mi propio jefe. Tengo mi empresa yo, hasta hoy, sigo trabajando con él, somos socios. Es una relación porque mi papá conocía el arquitecto este... (E46-V-NQN-INDUSTRIAL). 
Por último, entre los egresados varones de este grupo se destacan los jóvenes que estudiaron carreras terciarias y actualmente se desempeñan como docentes. En los inicios de sus recorridos la docencia no se presentó como una primera elección de estudios ni de trabajo, sino que una vez insertos laboralmente deciden volcar sus estudios a dicha área. Una cuestión para destacar es que dentro de la especialidad industrial son varios los casos que luego de fluctuar entre diversas carreras universitarias, los jóvenes articulan el dictado de clases con una formación en docencia. Un ejemplo es Federico quien estudió primero Ingeniería en Electrónica y después abandonó para estudiar un profesorado. Federico se había egresado como Técnico en Equipos e Instalaciones Electromecánica de una escuela secundaria de la Provincia de Buenos Aires. Su primera experiencia laboral había sido una pasantía a través de la escuela. Hasta el segundo tiempo tuvo por varios empleos, siempre relacionados con su especialidad (mantenimiento de máquinas en diversas fábricas de la rama industrial), hasta que se inició como docente, en lo que trabajaba hasta el presente:

Estoy dando clases de ajuste, herrería y soldadura. Y después, estoy dando clases de dibujo técnico y de electricidad. [...] 2016 seguí estudiando el profesorado. Ya lo tenía por la mitad, no tenía sentido dejarlo (E04-V-PBA-INDUSTRIAL).

En síntesis, en este grupo de jóvenes egresadas y egresados que lograron integrar su formación postsecundaria con la especialidad de su título técnico y obtuvieron una inserción laboral vinculada con esa formación se caracteriza por la heterogeneidad de tipos de formación, la multiplicidad de experiencias educativas (tienen diversas formaciones, aunque logren sólo finalizar una) y diversidad de experiencias laborales que en algunos casos se fueron hilvanando hasta alcanzar el trabajo actual. En algunos casos los empleos son de inserción profesional, en otros son trabajos informales, precarios y familiares (en el caso de los varones de especialidad agraria) así como emprendimientos propios. También se destacó que las mujeres son las que mayoritariamente continuaron y finalizaron carreras universitarias, contrario a los varones donde varios iniciaron carreras universitarias que no pudieron terminar. Sin embargo, se dan similitudes y no tantas diferencias: entre mujeres y varones una posibilidad fue el trabajo independiente y aquel en ámbito educativo (docencia). Los motivos, deseos y recorridos que los llevaron a esa inserción fueron distintos, pero también estas características (emprendedores y docentes) dan cuenta de lo que habilita el titulo técnico de nivel medio en los y las jóvenes, así como la disponibilidad de la oferta laboral que tienen en las distintas jurisdicciones analizadas.

\section{Consideraciones Finales}

A partir de la reconstrucción de las biográficas relevadas entre los egresados y las egresadas se identifican infinitas oraciones (biografías) que constituyen un sistema estructurado que da cuenta de este concepto de gramática (MIRANDA; CORICA, 2018). Es decir, una gramática de la juventud que estudio en escuelas técnicas, donde más allá de las diferencias encontradas aparecen espacios y ámbitos que contextualizan y determinan las experiencias juveniles en como también sus formas de acción (agencia) de los y las jóvenes sobre estas estructuras y determinaciones. Y, en este sentido, y considerando la totalidad del universo de jóvenes entrevistados (considerando todas las tipologías identificadas), el actuar de los y las jóvenes, como individuos y como grupo social en las estructuras e instituciones recorridas aparece una forma de responder a los desafíos que les va presentando la sociedad en diferentes contextos históricos durante sus trayectorias y recorridos biográficos particular de los egresados de escuelas técnicas. Con eso se quiere destacar la distinción y especificidad que da esta modalidad educativa en cuanto habilidades y conocimientos para "moverse en la vida" y en el mundo del trabajo. 
Ahora bien, también se identifica diversidad de recorridos, algunos más y otros menos vinculados educativa y laboralmente con la especialidad técnicas realizada, que da cuenta de que los procesos de inserción laboral son desiguales y reproductivitas. Pero también se identifica que la normativización de las trayectorias y desarrollo de los vínculos educación y trabajo en base a construcciones hegemónicas van conformando senderos a transitar socialmente convalidados, aunque no completamente legítimos, que entran en pugna con intereses y deseos de los propios sujetos.

En este sentido, se constata que no existen elementos de carácter económico universales a priori que detenten en la educación herramientas para los procesos de enclasamiento que se encuentran fuera de las condiciones contextuales y de las particularidades etarias y de género. Con las dificultades en la construcción de las trayectorias y las diversas condiciones con que los sujetos pueden desenvolverse en relación con su posición en la estructura y sus diversos capitales, están van a ser reflejo de la capacidad de agencia con que los diversos grupos o clases sociales detentan frente a los "éxitos" y "fracasos" de los recorridos individualizados, resultando de suma pertinencia la capacidad de intervención pública sobre los condicionantes y capitales situados.

En este sentido, cabe mencionar como la necesidad de elaboración de políticas públicas que definan y orienten carreras postsecundarias en las diversas regiones habilitan a la continuidad en sus localidades de estos/as egresados/as. El peso que tiene el titulo secundario y su orientación se pone en valor dentro de entramados productivos que potencien las oportunidades laborales. A su vez, y en relación con la elección de las orientaciones, cabe preguntarse: ¿ son los patrones de género tradicionales un factor de segmentación informal que impacta en la desigual matricula educativa? Si se tiene en cuenta que la muestra se compone de un mismo rango etario dentro de una temporalidad situada, para la tipología I el peso del origen de clase o social se encuentra en su mayoría compuesto por sectores medios. No obstante, cabe mencionar como las diferencias de género al momento de insertarse laboralmente, muestra que las jóvenes han tendido a una mayor extensión de su trayectoria formativa, lo cual pone en cuestión como, dentro de una territorialidad y temporalidad situada para un mismo sector social, las diferencias de género son subyacentes. Así como el registro de mayores actividades sociales y de cuidado entre las trayectorias de las mujeres. En cambio, entre los varones los emprendimientos propios y los trabajos vinculados con el entramado productivo local son las experiencias laborales más destacadas.

En este sentido, la disponibilidad de la oferta laboral que hay en las distintas jurisdicciones analizadas y el aporte por parte de la familia son dos elementos relevantes en la construcción de las trayectorias. En este marco se destaca la importancia que adquiere la posibilidad de inserción en la docencia en escuelas técnicas, en algunos casos las mismas de las cuales egresaron los jóvenes. El ámbito educativo como espacio oferente de puestos de trabajos es especialmente relevante en las jurisdicciones Salta y Neuquén.

Ahora bien, también la especialidad configura recorridos distintos. Entre los relatos de los egresados de la especialidad industrial existe una mayor certidumbre en cuanto a las posibilidades de ingreso al mercado laboral en un puesto vinculado con la orientación educativa recibida. En este grupo de jóvenes es donde se concentraron los casos de inserciones laborales afines a los conocimientos recibidos en la escuela secundaria técnica y donde se destacan que recibieron herramientas para insertarse laboralmente y/o realizar su propio emprendimiento una vez egresados del nivel medio. Mientras que los egresados de la especialidad Servicios la afinidad y/o adecuación de la inserción laboral se dio en aquellos que continuaron estudios postsecundarios, especialmente los que continúan carreras universitarias. En este sentido, esta especialidad, Servicios, se identificaron los casos que los egresados que obtuvieron empleos afines en esta especialidad, pero donde requería mayores credenciales educativas, es decir puestos de 
trabajos más profesionalizados. En cambio, la especialidad agropecuaria está fuertemente influenciada por el entorno familiar y cuestiones de género que dificultan o habilitan la inserción en ámbitos afines.

Por otro lado, el análisis de la reconstrucción de las trayectorias realizadas da cuenta que los procesos de inserción laboral se alargan en el tiempo, inclusive más de diez años post-egreso. Esto se da por diversos motivos:

1. La existencia de simultánea de realización de distintas carreras (universitarias y terciarias o terciarias y cursos de formación profesional);

2. La combinación de la actividad educativa y laboral al final de la cursada de las carreras de nivel superior;

3. Los cambios de trabajos; $y$

4. Las situaciones personales: ser madre, casarse o mudarse de ciudad. Todos estos elementos que se identificaron en todas las tipologías dan cuenta de las decisiones y elecciones que van considerando los y las jóvenes egresados en sus recorridos y, que muchas veces son independientes de un accionar racional económico como siempre se piensa.

Por último, la evidencia empírica relevada da cuenta de que la afinidad y la integralidad se va construyendo a lo largo del proceso de inserción laboral, y que más allá de que estos procesos se fueron dando en la combinación de los distintos elementos señalados, la afinidad aparece en los conocimientos aplicados y no sólo en un puesto laboral especifico. Así como la integralidad no es única, sino que puede ser que a lo largo de la trayectoria haya elementos integrales a la especialidad del título secundario técnico obtenido.

\section{Contribuición de los Autores}

Problematización: Corica A y Alfredo M; Conceptualización: Corica A y Alfredo M; Metodología, Corica A y Alfredo M; Análisis: Corica A y Alfredo M; Redacción: Corica A y Alfredo M.

\section{Notas}

1. La aplicación de la entrevista retrospectiva fue realizada por equipos locales de investigación. Por medio de la capacitación a las personas encargadas de realizar las entrevistas (cabe mencionar que las mismas eran estudiantes de grado y/o postgrado al momento de efectuar el campo), la implementación de estas se pauto para llevarse a cabo luego del contacto con los/as egresadas. Así, las entrevistas contaron con una extensión entre 40 a 90 minutos y fueron llevadas en los diversos lugares previamente coordinados con los entrevistados.

\section{Referencias}

BAUDELOT, C.; LECLERCQ, F. (dirs). Los efectos de la educación. Buenos Aires: Del Estante Editorial, 2008. 365 p. ISBN: 978-987-1335-15-2. 
BÉDUWÉ, C.; ESPINASSE, J.; VINCENS, J. Speciality of work and wages on recruitment the occupation vs the competency-based approach, Les Notes du LIRHE. Toulouse: LIRHE/Université des Sciences Socials de Toulouse, 2005. p. 1-29.

BENDIT, R.; HAHN-BLEIBTREU, M. (eds.). Youth transitions: processes of social inclusion and patterns of vulnerability in a globalised world. Opladen: Verlag Barbara Budrich. 2008. https://doi.org/10.2307/j. ctvdf09qg

BORRAS, I.; LEGAY, A.; ROMANI, C. Les choix d’orientation face à l’emploi. Bref, Marseille, n. 258, nov. 2008. BOWLES, S.; GINTIS, H. La instrucción escolar en la América capitalista. La reforma educativa y las contradicciones de la vida económica. Ciudad de México: Siglo XXI Editores, 1986.

CASAL, J. et al. Itinerarios y trayectorias: una perspectiva de la transición de la escuela al trabajo. Trayectorias: Revista de Ciencias Sociales, Monterrey, año VIII, n. 22, p. 9-20, 2006.

COLL, J. P. La relación entre educación y empleo en Europa. Papers: Revista de Sociologia, Orono, v. 96, n. 4, p. 1047-1073, 2011. https:// doi.org/10.5565/rev/papers/v96n4.168

CUERVO, H.; WYN, J. Reflections on the use of spatial and relational metaphors in youth Studies. Journal of Youth Studies, London, v. 17, v. 7, p. 901-915, 2014. https://doi.org/10.1080/13676261.2013.878796

ELDER, G. H. Time, human agency, and social change: perspectives on the life course. Social Psychology Quarterly, New York, v. 57, n. 1, p. 4-15, 1994. https://doi.org/10.2307/2786971

FILMUS, D. et al. Cada vez más necesaria, cada vez más insuficiente: escuela media y mercado de trabajo en épocas de globalización. Buenos Aires: Santillana, 2001.

FURLONG, A. (ed.). Handbook of young and young adulthood. New Perspectives and agendas. New York: Routledge International, 2009.

GLASER, B.; STRAUSS, A. The discovery of grounded theory. Chicago: Aldine Press, 1967.

GIL CALVO, E. Trayectorias y transiciones: ¿Qué rumbos? Revista de Estudios de Juventud, Madrid, n. 87, p. 15-30, 2009. ISSN-e 0211-4364.

KESSLER, G. Controversias sobre la desigualdad: Argentina 2003-2013. Buenos Aires: Fondo de Cultura Económica, 2014.

KEOGAN, L.; CALÁ, C.; BELMARTINO, A. Perfiles sectoriales de especialización productiva en las provincias argentinas: distribución intersectorial del empleo entre 1996 y 2014. Regional and Sectoral Economic Studies, Santiago de Compostela, v. 20, n. 1, p. 59-80, 2020.

MIRANDA, A. (coord.). La construcción de trayectorias laborales de los egresados de la escuela técnica: una mirada sobre la integralidad de la formación a una década de la Ley de Educación Técnico Profesional. Informe final. FONIETP, 2018.

MIRANDA, A.; CORICA, A. Gramáticas de la Juventud: reflexiones conceptuales a partir de estudios longitudinales en Argentina. In: CORICA, A. et al. (comps.). Entre la educación y el trabajo: la construcción cotidiana de las desigualdades juveniles en América Latina. Ciudad Autónoma de Buenos Aires: CLACSO, 2018. 
PÉREZ, P.; BUSSO, M. Juventudes, educación y trabajo. In: GIOVANNI, J.; SALVIA, A. (coords.). La Argentina en el siglo XXI: cómo somos, vivimos y convivimos en una sociedad desigual: Encuesta Nacional sobre la Estructura Social. Ciudad Autónoma de Buenos Aires: Siglo XXI Editores Argentina, 2018

PUJADAS MUÑOZ, J. J. El método biográfico. El uso de las historias de vida en ciencias sociales. Madrid: Centro de Investigaciones Sociológicas, 2002.

REYNOLDS, T. Black Neighborhoods' and 'Race.' Placed Identities in Youth Transition to Adulthoods. In: WYN J.; CAHILL H. (eds.). Handbook of children and youth studies. Singapore: Springer, 2015.

SAUTU, R. (comp.). El método biográfico. Buenos Aires: Editorial Lumiere, 2004.

WELLER, J. La problemática inserción laboral de los y las jóvenes. Santiago de Chile: United Nations Publications/División de Desarollo Económico, 2003. v. 28.

WILLIS, P. Aprendiendo a trabajar: como los chicos de la clase obrera consiguen trabajos de clase obrera. Madrid: AKAL Pensamiento Crítico, 1988.

WYN, J.; WOODMAN, D. Generation, youth and social change in Australia. In: Journal of Youth Studies, London, n. 9, n. 5, p. 495-514, 2006. https://doi.org/10.1080/13676260600805713

\section{Sobre los Autores}

Agustina Corica es doctora en Ciencias Sociales y Licenciada en Sociología de la Universidad de Buenos Aires (UBA). Magíster en Diseño y Gestión en Políticas y Programas Sociales. Investigadora Adjunta del Consejo Nacional de Investigaciones Científicas y Técnicas e Investigadora Principal en Universidad Pedagógica Nacional (UNIPE). Coordinadora Académica del Programa de Investigaciones de Juventud de la FLACSO Argentina. Docente en la Universidad del Salvador (USAL) y en la Universidad de Buenos Aires (UBA).

Miguel Alfredo es doctorando en Ciencias Sociales de la Universidad de Buenos Aires. Magister en Ciencias Sociales del Trabajo y Licenciado en Sociología. Becario doctoral por temas estratégicos del Consejo Nacional de Investigaciones Científicas y Técnicas. Diplomado Superior en Estudios y Políticas de Juventud en América Latina. Investigador asistente del Programa Estudios y Relaciones del Trabajo/FLACSO Argentina y Programa Juventud/FLACSO Argentina. Se ha desempeñado como docente en USAL y UNLaM.

Recibido: 26 Oct. 2019

Aceptado: 02 Oct. 2020 


\section{Anexo Estadístico}

Muestra segmentada por establecimientos educativos intencional y no probabilística. Dicha selección corresponde a definiciones tradicionales de los estudios de la segmentación educativa (MIRANDA; CORICA, 2018). En este sentido, la misma se delimitaron por: i. localización espacial; ii. Infraestructura escolar y tipo de institución; iii. Origen socioeconómico.

Tabla 3. Composición demográfica de la muestra según escuela por jurisdicción. Año 2018

\begin{tabular}{|c|c|c|c|c|c|c|}
\hline \multirow{2}{*}{ Escuela } & \multirow{2}{*}{$\begin{array}{c}\text { Cantidad } \\
\text { de } \\
\text { entrevistas }\end{array}$} & \multicolumn{2}{|c|}{ Localización } & \multicolumn{2}{|c|}{ Institución } & \multirow{2}{*}{ Origen social } \\
\hline & & Jurisdicción & Tipo de barrio & Infraestructura & $\begin{array}{c}\text { Tamaño } \\
\text { matricula }\end{array}$ & \\
\hline 1 & 1 & CABA & Clase media & Muy bien & Mediana & Medio-medio \\
\hline 2 & 4 & CABA & Clase media & Muy bien & Mediana & Medio-medio \\
\hline 3 & 1 & CABA & Clase media & Buena & Mediana & Medio-bajo \\
\hline 4 & 1 & CABA & Clase media & Buena & Chica & Medio-bajo \\
\hline 5 & 1 & CABA & Clase media & Buena & Mediana & Medio-bajo \\
\hline 6 & 1 & CABA & Clase media & Excelente & Grande & Medio-alto \\
\hline 7 & 2 & CABA & Clase media & Excelente & Grande & Medio-alto \\
\hline 8 & 1 & CABA & Clase media & Muy bien & Grande & Medio-medio \\
\hline 9 & 1 & CABA & Clase media & Muy bien & Mediana & Medio-medio \\
\hline 10 & 1 & PBA & Clase media & Buena & Mediana & Medio-bajo \\
\hline 11 & 1 & PBA & Clase media & Muy bien & Mediana & Medio-medio \\
\hline 12 & 5 & PBA & Clase media & Muy bien & Chica & Medio-medio \\
\hline 13 & 5 & PBA & Zona rural & Muy bien & Chica & Medio-medio \\
\hline 14 & 1 & PBA & Clase media & Excelente & Chica & Medio-medio \\
\hline 15 & 2 & PBA & Clase media & Buena & Mediana & Medio-medio \\
\hline 16 & 1 & PBA & Clase media & Muy bien & Chica & Medio-alto \\
\hline 17 & 5 & NQN & Clase media & Muy bien & Chica & Medio-medio \\
\hline 18 & 1 & NQN & Clase media & Muy bien & Mediana & Medio-alto \\
\hline 19 & 3 & NQN & Barrio relegado & Buena & Mediana & Medio-bajo \\
\hline 20 & 11 & NQN & Clase media & Buena & Mediana & Medio-medio \\
\hline 21 & 2 & SLA & Clase media & Excelente & Mediana & Medio-medio \\
\hline 22 & 1 & SLA & Clase media & Mala & Chica & Medio-alto \\
\hline 23 & 2 & SLA & Clase media alta & Buena & Mediana & Medio-medio \\
\hline 24 & 6 & SLA & Zona semiurbana & Buena & Chica & Medio-bajo \\
\hline 25 & 1 & SLA & Clase media & Buena & - & Medio-medio \\
\hline 26 & 1 & SLA & Clase media & Buena & - & Medio-bajo \\
\hline 27 & 1 & SLA & Clase media & Mala & - & Medio-bajo \\
\hline 28 & 4 & SLA & Barrio relegado & Mala & Chica & Medio-bajo \\
\hline 29 & 2 & SLA & Clase media alta & Buena & Chica & Bajo \\
\hline
\end{tabular}

Fuente: Elaboración propia en base Miranda (2018). 